cells were depleted. From the levels of metabolite concentrations it was not clear if the cause of the decrease in the energy-rich phosphates and the increased anaerobic glycolysis could be found in a specific disturbance of oxidative phosphorylization-for example, ferment inhibition-or in insufficiency of oxygen supply. The latter seemed the most likely explanation.

On the basis of these investigations it was concluded that the twin aims of the therapy of cerebral oedema were the removal of increased extracellular fluid in the white matter and restitution of normal vascular permeability. It was also pointed out that oxygen deficiency, due to respiratory embarrassment, shock, or hyperthermia was likely to cause irreversible damage to oedematous tissue more readily than to normal tissue.

The preceding two papers were discussed by $W$. Wesemann (Giessen) and A. R. Taylor (Belfast).

\section{DIRECT MEASUREMENT OF INTRACRANIAL PRESSURE AFTER OPERATION}

E. MARKAKIS, E. BUES, and A. SPRING (Kiel) described a method of direct measurement of intracranial pressure by means of a transducer incorporating a new semiconductor element. The transducer consisted of a disk $1 \mathrm{~mm}$ thick and 3 to $6 \mathrm{~mm}$ in width. Transducers, which were encased in an ultra-thin capsule of beryllium-copper, were coated with silastic rubber as protection from body tissues. Implantation up to four weeks was possible and the transducers were designed to be compatible with all types of standard strain-gauge instrumentation. The results pointed to the importance of direct pressure measurements in clinical practice by continuous monitoring. Increased intracranial pressure could thereby be detected early and treatment started immediately.

\section{HYPOTHALAMIC LESIONS IN HEAD INJURY}

C. S. TREIP (Cambridge) reported the histological examination of the hypothalamus in 16 cases of fatal head injury, some of whom showed clinical evidence of hypothalamic-pituitary disturbance, such as diabetes insipidus, electrolyte imbalance, and persistent hypothermia. The principal lesions found were:

1. Haemorrhage into and disruption of the supraoptic nucleus, due probably to shearing strains imposed by sudden movements of the brain (Treip, 1970). The end result of this acute lesion, in long survivors, was atrophy with loss of magnocellular (neurosecretory) neurones. As bilateral involvement of the supraoptic nucleus was very rare, diabetes insipidus of any severity was correspondingly uncommon (one case in this series). The paraventricular nucleus was less directly involved by trauma than the supraoptic nucleus.

2. Acute infarction of the infundibulum, due probably to interruption or spasm of the upper branches of the superior hypophysial artery ring. This lesion, if in the midline, could also lead to diabetes insipidus. Bilateral infarction of the ventromedial nuclei occasionally occurred in association with severe electrolyte imbalance (hypernatraemia); this might have been related to disturbance of production of corticotrophin releasing factor in the infundibulum.
3. Widespread damage to the periventricular grey matter of the third ventricle was in three cases associated with persistent hypothermia. These clinicopathological correlations might be of assistance in the recognition and study of hypothalamic injury during life.

\section{REFERENCE}

Treip, C. S. (1970). In Symposium on the Pathology o, Trauma, Royal College of Pathologists (in press).

DIAGNOSIS AND TREATMENT OF SPINAL ANGIOMAS

H. W. PIA (Giessen) described a total of 88 angiomas in 70 patients which had demonstrated that $60 \%$ of angiomas were solitary and $40 \%$ were complex, consisting of vertebral-extradural angiomas, more rarely extradural and intradural angiomas, and occasionally the simultaneous occurrence of angiomas and angioblastomas.

Epidural angiomas, mostly cavernous and racemose, were more frequent than usually thought. The most frequent intradural angioma was the subarachnoid arteriovenous cirsoid type fed by the dorsal radicular arteries.

As in the case of cerebral angiomas, the most important and constant disturbance was the local reduction of blood flow due to the A-V shunt, a spinal 'steal syndrome'. Primary compression with spinal block was unusual, even with large vessels but secondary compression of the cord was found in $50 \%$ of cases. Adhesive and cystic arachnoiditis probably caused by unsuspected haemorrhages, were the usual cause of spinal block in these cases. In the intradural lesions, however, early compressive symptoms predominated.

The difficulties of diagnosis in this condition were very apparent. In an earlier report of 54 cases, which was referred to, no correct diagnosis had been made in cases of extradural angioma and the suspicion was expressed in only three cases of intradural angioma. In more than half of the cases described acute spinal apoplexy and a relapsing apoplectic course were seen and were regarded as strongly suspicious of a spinal angioma.

The special diagnostic procedures indicated in this condition were described with particular reference to spinal ossovenography before myelography and in certain selected cases angiography according to the technique of Djindjian and Di Chiro. Myelography, however, had remained indispensable in this diagnosis.

The radical removal of these lesions, wherever possible, was recommended, and the author's experience confirmed that, although this was only possible in the past in about half the cases, at present two-thirds yielded to this method of treatment. He recommended the use of magnification, bipolar coagulation, and microsurgical instruments. He was not in favour of occlusion of feeding vessels without excision of the main lesion because 'the demand for blood' of the angioma persisted and the reduced blood flow might act to the detriment of the cord.

SPONTANEOUS HAEMORRHAGE IN THE SPINAL CANAL

TH. SCHAAKE and E.-R. SCHÄFER (Göttingen) described 
three cases of spontaneous haemorrhage in the spinal canal, and the history of this condition, as it appears in the literature, was presented. In none of the cases did the haemorrhage result from an angioma; in two cases the bleeding was subdural and in one case extradural. The authors felt that slight trauma, which was usually held responsible for these episodes, was probably coincidental and that the origin, not only of extradural bleeding but also of subdural bleeding, was probably from a venous anomaly. One of their cases was due to a defect of blood coagulation due to anticoagulant therapy. In two cases spinal cord compression was severe and operation was undertaken later than would otherwise have been the case if the patient had initially been seen in a Department of Medical or Surgical Neurology.

\section{REGULATION OF SPINAL BLOUD FLOW UNDER} PHYSIOLOGICAL AND PATHOLOGICAL CONDITIONS

H. PAlleske, H.-D. herrmanN, and F. LOEW (Homburg/ Saar) investigated the regulation of spinal cord blood flow in $\mathbf{4 8}$ dwarf pigs by applying a heat clearance probe to the lumbar cord at laminectomy. Simultaneously a similar probe was placed on the surface of the brain to correlate the two readings. The aorta was also exposed so that the blood supply to the lower part of the body could be occluded when required. They came to the conclusion that the regulation of the blood flow of the spinal cord was very much the same as in the brain and that the different reaction to vascular compression could be attributed to differences in the vascular pattern in the spinal cord.

This paper was then discussed by K. Piscol (Heidelberg), R. Wüllenweber and F. K. Schröder (Bonn).

\section{MYELOPATHY IN CERVICAL SPONDYLOSIS: SURGICAL} TREATMENT USING A POSTEROLATERAL APPROACH

ANTONY JEFFERSON (Sheffield) reported a posterolateral approach for the surgical treatment of myelopathy with cervical spondylosis which had been routinely employed for the past five years. For three years, a wide laminectomy (usually from $\mathrm{C} 3$ to $\mathrm{C} 6$ inclusive) had been combined with the removal of the cartilaginous and osteophytic disc lesion. The prone cerebellar position was used. The instruments were simple. As well as the vertical incision required for the laminectomy, a transverse incision through skin and muscle was made to allow the correct trajectory of the instruments. Laminectomy was considered essential because ligamentum flavum, indenting the theca, could often be displayed on myelography. Myelograms indicating the pre- and post-operative appearances were shown, as well as a film of a recently operated patient. Among the first 50 patients, none had been worse post-operatively, $10 \%$ were unchanged, $22 \%$ had improved marginally but were still unable to work, while $68 \%$ had resumed work $(39 \%$ of this last category had a negligible disability). Analysis of a patient's clinical state at the time of operation indicated that the best results followed when the pre-operative handicaps were not gross, when hyperreflexia was not excessive, and when at least one plantar response remained flexor. After both plantar responses had become extensor, operation- $\bar{Z}$ if contemplated-should be performed as soon as con- $\mathbb{D}$ servative measures had proved themselves ineffective. It was suggested that this technique appeared both useful and safe.

\section{PROPERTIES OF SOMATOSENSORY NEURONES IN THE} HUMAN THALAMUS

A. J. MCCOMAS, P. WILSON, J. MARTIN-RODRIGUEZ, C. WALLACE, and JOHN HANKINSON (Newcastle upon Tyne) had studied the electrophysiological properties of thalamic neurones during the course of stereotaxic surgery for various movement disorders, particularly $\overline{\bar{c}}$ Parkinsonism, and also for intractable pain. Altogether $\frac{\mathcal{S}}{\partial}$ 57 patients were investigated, of whom the majority were $\underset{\Omega}{\mathbb{D}}$ fully conscious at the time of recording; in these patients a total of 83 tracks were made with tungsten microelectrodes.

The electrodes were directed through a posterior burr $\overrightarrow{-}$ hole towards a target in the ventralis oralis posterior $\vec{\omega}$ nucleus, $2 \mathrm{~mm}$ above the intercommisural axis at the junction of its middle and posterior thirds. The correct $\bar{\partial}$ localization of this target was greatly facilitated by a? detailed survey of neurones in the somatosensory region $\omega$ lying posteriorly (presumably $N$. ventralis caudalis and $N$. ventralis intermedius). In the anteroposterior axis was possible to delineate the anterior border of thiss $\vec{T}$ somatic area, and also the interface between $\mathrm{N}$. ventraiss oralis and the internal capsule, with considerable accuragy (probably to $0.5 \mathrm{~mm}$ ). In the transverse axis the evokg $\rightarrow$ somatosensory potentials enabled seven degrees $\forall \mathrm{f}$ laterality to be identified. Thus, as the electrode was? advanced anteriorly, successively more lateral tracks encountered the following sequences of topographick. 1 은 representation:

Most medial track: No evoked potentials Face (responses barely detectable) Face

Face $\rightarrow$ hand

Hand $\rightarrow$ face $\rightarrow$ hand

Hand

Most lateral track: Foot

Since the anterior border of the somatosensory nuclei occupied about $9 \mathrm{~mm}$ in the transverse axis, the recognition of seven degrees of laterality (above) conferred an accuracy of not less than $1.5 \mathrm{~mm}$ in this dimension. For most patients the best site for the centre of the lesion (6 $\mathrm{mm}$ diameter) was a point $2 \mathrm{~mm}$ beyond the anterior border of the somatosensory region which representedo the face first and then the hand.

Of the 122 somatosensory neurones investigated to date, 86 responded to touching the skin, and 31 to movements of joints, on the contralateral side; a further fiveo units had high thresholds and could not be classified? satisfactorily. The joints most frequently concerned were the metacarpo-phalangeal ones and units responding to extension were most prevalent. The receptive fields of then 'cutaneous' units varied from $0.3 \mathrm{~cm}^{2}$ to $30 \mathrm{~cm}^{2}$ in size? in general, the units with the smallest fields were those 